\title{
Age Differences in Adults' Use of Referring Expressions
}

\author{
Petra Hendriks • Christina Englert • Ellis Wubs • \\ John Hoeks
}

\begin{abstract}
The aim of this article is to investigate whether choosing the appropriate referring expression requires taking into account the hearer's perspective, as is predicted under some versions of bidirectional Optimality Theory but is unexpected under other versions. We did this by comparing the results of 25 young and 25 elderly adults on an elicitation task based on eight different picture stories, and a comprehension task based on eight similar written stories. With respect to the elicitation task, we found that elderly adults produce pronouns significantly more often than young adults when referring to the old topic in the presence of a new topic. With respect to the comprehension task, no significant differences were found between elderly and young adults. These results support the hypothesis that speakers optimize bidirectionally and take into account hearers when selecting a referring expression. If the use of a pronoun will lead to an unintended interpretation by the hearer, the speaker will use an unambiguous definite noun phrase instead. Because elderly adults are more limited in their processing capacities, as is indicated by their smaller working memory capacity, as speakers they will not always be able to reason about the hearer's choices. As a result, they frequently produce non-recoverable pronouns.
\end{abstract}

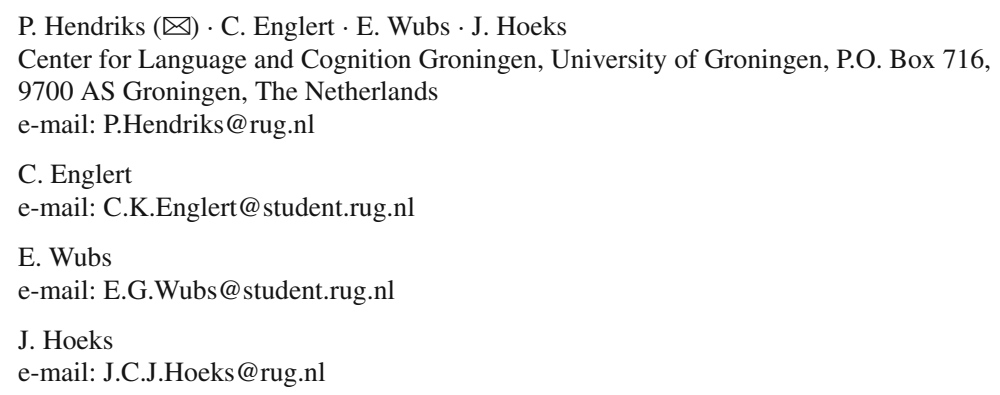


Keywords Bidirectional Optimality Theory - Discourse structure - Language comprehension $\cdot$ Language production $\cdot$ Referring expressions

\section{Introduction}

Speakers tend to use different types of referring expressions depending on the structure of the previous linguistic discourse. For example, they use pronouns for entities that are present in the immediate linguistic discourse, indefinite noun phrases (NPs) to introduce new entities into the linguistic discourse, and definite NPs if the entity has been mentioned before but is not the main focus of attention. Many studies relate this dependency of the use of referring expressions on discourse structure to the accessibility or topicality of the referent, or the givenness of the referent for the hearer (Ariel 1988, 1990; Givón 1993; Gundel et al. 1993). According to Gundel et al. (1993), givenness must be seen in terms of a hierarchy, or implicational scale. When speakers can use different forms to refer to the same thing, they will use the form on the scale that is just informative enough to allow the hearer to identify the intended referent, thus observing Grice's Maxim of Quantity (Grice 1975).

Based on the theoretical framework of Optimality Theory (Prince and Smolensky, 1993/2004), Blutner (2000) proposes a reduction and formalization of Grice's maxims of conversation which integrates the speaker perspective and the hearer perspective into a simultaneous optimization procedure. The resulting formal model is known as bidirectional Optimality Theory (bi-OT). According to bi-OT, hearers choose the best meaning for a heard form, thereby taking into account the speaker. This can be modeled as a sequential process (cf. Hendriks et al. 2007): If a meaning is selected when optimizing from a heard form to its optimal interpretation, but this selected meaning does not yield the heard form again when the direction of optimization is reversed, this meaning is blocked as a possible interpretation. Conversely, speakers choose the best (usually, most economical) form for the intended meaning, thereby taking into account the hearer. If the selected form does not yield the intended meaning again in comprehension, this form is blocked as a possible form in production. As a result, speakers are just informative enough to get the message across. Obviously, taking into account one's own perspective as well as the opposite perspective in communication is more complex than merely taking into account one's own perspective. For this reason, it is not surprising that children have difficulties with linguistic tasks that seem to require bidirectional optimization, that is, tasks that require language users to also take into account the opposite perspective. Children's late comprehension problems with indefinite NPs and object pronouns, sometimes lasting until the age of 7 or even longer, can thus be explained as resulting from their inability to optimize bidirectionally (de Hoop and Krämer 2005/2006; Hendriks and Spenader 2004, 2005/2006).

However, two objections can be raised against a bi-OT explanation of children's late comprehension problems. First of all, it seems difficult to distinguish empirically between a bi-OT explanation and other non-syntactic explanations. Consider children's problems with the comprehension of object pronouns such as him. As is 
well-known, children incorrectly allow him in the sentence Bert washed him to refer back to the subject Bert until the age of 6 or 7 (e.g., Chien and Wexler 1990). Hendriks and Spenader (2004, 2005/2006) argue that, in order to correctly interpret object pronouns such as him as not referring to the subject, children must learn to consider the speaker's perspective. Only if a hearer is able to consider the alternative but unheard reflexive form himself, which unambiguously refers back to the subject in the sentence Bert washed himself, can the hearer block the interpretation according to which him refers back to the subject Bert. Reinhart (2004, to appear), on the other hand, claims that children possess the required grammatical and pragmatic knowledge but simply lack sufficient working memory capacity for the parser to perform the required comparison between the relevant forms and their meanings. But note that, because bidirectional optimization is more complex than unidirectional optimization and requires the simultaneous activation of forms as well as meanings, a bi-OT explanation would be contingent on sufficient working memory capacity too. Another possibility, advocated by Thornton and Wexler (1999), is that children lack the relevant pragmatic knowledge to distinguish exceptional cases from standard cases. Thornton and Wexler argue that this is why for children Bert washed him is ambiguous: They do not yet know when they are dealing with a standard case where him cannot refer to the subject, and when they are dealing with an exceptional case such as Everyone here admires John. Mary admires John, Sue admires him, and John admires him too. Here, even adults allow him to refer back to the subject. It is not easy to distinguish between these different explanations for children's late comprehension problems (i.e., Hendriks and Spenader's bi-OT explanation, Reinhart's processing explanation, and Thornton and Wexler's pragmatic explanation) by looking at children's comprehension data only.

One way to tease apart cognitive factors such as task complexity, working memory capacity and speed of processing from lack of grammatical or pragmatic knowledge is by studying elderly adults. We may assume that elderly adults possess the required grammatical and pragmatic knowledge to select and interpret referring expressions. If their linguistic performance is defective, this must be due to cognitive factors such as working memory capacity or speed of processing. Earlier studies have shown that with age working memory capacity decreases (Wingfield et al. 1998). Moreover, there seems to be a clear correlation between working memory capacity, age, and the way quantifier ambiguities are resolved (Kemtes and Kemper 1999), suggesting that some aspects of linguistic performance indeed change with age.

A second objection against a bi-OT explanation of children's late comprehension problems is that it assumes the adult interpretation to be obtained through bidirectional optimization. Blutner and Zeevat (2004) point out that an online view of bidirectional optimization gives rise to several problems and suggest that bidirectional optimization must be seen in an evolutionary perspective. ${ }^{1}$ When two forms and two meanings are available, language users will pair an optimal form to an optimal meaning, and a suboptimal form to a suboptimal meaning, by applying pragmatic principles of language use. Eventually, these form-meaning pairs become conventionalized (or 'fossilize', in

\footnotetext{
1 But see Blutner et al. (2006) and Bouma (2008) for possible solutions to the problem of overgeneration and the $\mathrm{Rad} / \mathrm{Rat}$ problem in syntax, respectively.
} 
the terminology of Blutner 2007), until one form is used exclusively for the one, and the other form for the other meaning. On Blutner and Zeevat's view, form-meaning pairs that have thus been determined by bidirectional optimization constitute fixed relations to a learner who sets out acquiring the language, and no learner or user of the language needs to perform a bidirectional optimization computation for any form-meaning pair she encounters. For the interpretation of object pronouns mentioned above, for example, such a fossilized form-meaning pair could consist of an object pronoun (the form) and disjoint reference with respect to the subject (the meaning), which boils down to the well-known Principle B from Binding Theory (Pronouns must be free locally, i.e., object pronouns must be disjoint to the local subject). ${ }^{2}$ But on the basis of adults' linguistic performance with respect to sentence-internal phenomena such as the interpretation of object pronouns, it is impossible to tell whether the form-meaning pairs in their grammar have arisen as a result of fossilization (as Blutner and Zeevat argue), or as a result of online bidirectional optimization which may have become automatic because of its frequent use in the same fixed sentence-internal context (Hendriks et al. 2007).

To determine whether adults have to compute at least some form-meaning pairs anew, we should look at discourse phenomena. Here, it is less likely that the association between form and meaning has become automatic, because discourse context is highly variable. For example, we may look at referring subjects such as he in He washed Bert, which are not dependent on material within the same sentence for their use but rather depend on the previous discourse. If mature language users take into account the opposite perspective in communication, we should be able to see effects of online bidirectional optimization in their use of referring subjects.

The aim of this article is to investigate whether choosing the appropriate referring expression requires a speaker to take into account the hearer's perspective. We focus on the use of referring subjects by elderly participants for the two reasons mentioned above. The formal bidirectional Optimality Theory model and the predictions it makes are discussed in Sect. 2. If speakers take into account hearers, the selection of a referring expression is expected to require additional linguistic processing and additional working memory capacity. We investigate this issue by presenting young and elderly adults with picture stories and asking them to tell a story on the basis of these pictures. This elicited production experiment is discussed in Sect. 3. In a second study (a comprehension experiment discussed in Sect. 4), we used similar stories, but now based on a linguistic discourse rather than on a series of pictures. We then asked comprehension questions about these discourses. Section 5 provides a general discussion on the basis of a comparison between the results of the production experiment and the comprehension experiment, and Sect. 6 presents our conclusions.

\footnotetext{
2 Note that the observation that children display knowledge of Principle A (Reflexives must be bound locally) from an early age on, whereas they continue to make comprehension errors with respect to its sister principle Principle B (Pronouns must be free locally) until the age of 6 or 7 (e.g., Chien and Wexler 1990) suggests that, at least for children, knowledge of Principle A and knowledge of Principle B are of a different type. This remains unexplained under Blutner and Zeevat's account.
} 


\section{A Formal Model of Pronoun Production and Comprehension}

In this section, we introduce the formal model of bidirectional optimization and discuss the predictions the model yields with respect to referring subjects. These predictions will be tested in two studies, discussed in Sects. 3 and 4, respectively.

Bi-OT combines the perspective of the speaker with the perspective of the hearer and can be defined as follows:

(1) Bidirectional optimization (adapted from Blutner 2000):

A form-meaning pair $<\mathrm{f}, \mathrm{m}>$ is bidirectionally optimal iff:

a. there is no other bidirectionally optimal pair $<\mathrm{f}^{\prime}, \mathrm{m}>$ such that $<\mathrm{f}^{\prime}, \mathrm{m}>$ is more harmonic than $<\mathrm{f}, \mathrm{m}>$.

b. there is no other bidirectionally optimal pair $<$ f,m' $>$ such that $<\mathrm{f}$,m' $>$ is more harmonic than $<\mathrm{f}, \mathrm{m}>$.

The term 'harmonic' in this definition indicates how well an output candidate satisfies the constraints of the grammar. Under this definition, forms and meanings are not considered separately. Instead, optimization occurs over pairs consisting of forms and their corresponding meanings. A form-meaning pair is an optimal pair if there is no pair with a better form or a better meaning. Only optimal pairs are realized in language. Such optimal pairs block all other pairs in the same competition.

The relative harmony of form-meaning pairs is determined by the constraints of the grammar. A constraint sub-hierarchy playing a role in the choice of referring expressions is the universal constraint sub-hierarchy REFERENTIAL ECONOMY (cf. Burzio 1998). This constraint sub-hierarchy reflects the view that expressions with less referential content are preferred over expressions with more referential content and has the effect that reflexives are preferred over pronouns, and pronouns are preferred over R-expressions (i.e., full NPs). Because we are concerned with subjects only, and reflexives cannot occur in subject position in languages such as English and Dutch, only the two constraints AVOID R- EXPRESSIONS and AVOID PRONOUNS of this constraint sub-hierarchy are relevant for the present discussion. A third constraint that is relevant is the constraint PRONOUNS REFER TO THE TOPIC. This constraint expresses a preference for pronouns to be interpreted as the topic of the discourse, and is violated by any pronoun that refers to a non-topic. Adopting these three constraints, we can depict their interaction by means of an OT Tableau.

Given the choice between a pronoun and an R-expression as the form to be selected, and between a topic and a non-topic as the meaning to be selected, there are four logically possible form-meaning pairs. These pairs are listed in the first column of bidirectional optimization Tableau 1. Constraints in an OT Tableau are ordered from left to right in the first row, in order of descending strength. The linear order of the three constraints shows that AVOID R- EXPRESSIONS is stronger than AvOID PRONOUNS. The relative ranking of the constraint AvOID PRONOUNS and the constraint PRONOUNS REFER TO THE TOPIC is not relevant for the present analysis.

A crucial property of OT is the violability of constraints. Constraints are potentially conflicting and hence violable. If two constraints are in conflict, it is more important 
Tableau 1 Bidirectional optimization of referring subjects

\begin{tabular}{|c|c|c|c|c|}
\hline & & $\begin{array}{l}\text { AVOID } \\
\text { R- EXPRESSIONS }\end{array}$ & $\begin{array}{l}\text { AVOID } \\
\text { PRONOUNS }\end{array}$ & $\begin{array}{l}\text { PRONOUNS REFER } \\
\text { TO THE TOPIC }\end{array}$ \\
\hline \multirow[t]{3}{*}{$B$} & $<$ pronoun, topic $>$ & & $*$ & \\
\hline & $<$ pronoun, non-topic $>$ & & $*$ & $*$ \\
\hline & $<$ R-expression, topic $>$ & $*$ & & \\
\hline$B$ & $<$ R-expression, non-topic $>$ & $*$ & & \\
\hline
\end{tabular}

to satisfy the stronger constraint than it is to satisfy the weaker constraint. Because the first pair, < pronoun, topic >, violates only the constraint AVOID PRONOUNS, whereas all other pairs violate additional or stronger constraints, this first pair is a bidirectionally optimal pair according to the definition given in (1). This is marked by $\otimes$ in the Tableau. There is no other pair that satisfies the constraints better (i.e., that is more harmonic). As a result, this first pair blocks all other pairs with the same form but a less harmonic meaning (in this example, the second pair) and pairs with the same meaning but a less harmonic form (in this example, the third pair). Importantly, according to the definition given also the fourth pair $<$ R-expression, non-topic $>$ is bidirectionally optimal. It does not have any bidirectionally optimal competitors with a more harmonic form or a more harmonic meaning. The second pair is not bidirectionally optimal, and the first pair does not compete with the fourth pair because they have no form or meaning in common. As a result of this bi-OT competition, pronouns are predicted to be used for topics and vice versa, and R-expressions are predicted to be used for non-topics and vice versa.

Whereas adults may optimize bidirectionally and produce the predicted pattern of pronouns and R-expressions, it has been argued that children until at least the age of 6 are not yet able to optimize bidirectionally (de Hoop and Krämer 2005/2006; Hendriks and Spenader 2004, 2005/2006). Instead, they optimize unidirectionally, without taking into account the opposite perspective. In comprehension, they optimize from a given form to the optimal meaning for this form, without considering any alternative forms the speaker could have used. In production, they optimize from an intended meaning to the optimal form to express this meaning, without considering any alternative meanings the hearer may assign to the expressed form. Because bidirectional optimization is more complex and hence requires more cognitive resources than unidirectional optimization, it is conceivable that under certain circumstances also adults may fail to optimize bidirectionally and produce a unidirectionally optimal output instead.

Now what are the predictions the proposed constraints yield for unidirectional optimization? Do these predictions deviate from the predictions of the bi-OT model? Let us first look at comprehension. Given a subject pronoun, the optimal meaning expressed by this form is reference to the topic. Reference to a non-topic would violate the constraint PRONOUNS REFER TO THE TOPIC. So subject pronouns are interpreted the same under a unidirectional and a bidirectional mode of optimization. Given an R-expression, however, both reference to the topic and reference to a non-topic are optimal meanings. They violate and satisfy the same constraints. 
As a result, R-expressions receive a different interpretation depending on whether they are interpreted bidirectionally or unidirectionally.

Also with respect to production, we predict differences between a bidirectional and a unidirectional mode of optimization. If we wish to refer to the topic, the optimal form is a pronoun. However, if we wish to refer to a non-topic, the optimal form also is a pronoun. Because the constraint AVOID R- EXPRESSIONS is stronger than the constraint PRONOUNS REFER TO THE TOPIC, a pronoun is the optimal form for referring to a topic as well as to a non-topic. By using a pronoun for referring to a non-topic, the weaker constraint PRONOUNS REFER TO THE TOPIC is violated, but this is tolerated because this is the only way to satisfy the stronger constraint AVOID R- EXPRESSIONS. So again we have a different output depending on the mode of optimization. Under the unidirectional mode of optimization, subject pronouns are overused since they are also used for referring to a non-topic. Under the bidirectional mode of optimization, in contrast, subject pronouns are only used for referring to the topic, not for referring to a non-topic.

If a language user fails to optimize bidirectionally, our bi-OT analysis thus predicts problems in comprehension (with respect to R-expressions) as well as problems in production (with respect to pronouns). Consequently, the predictions of our bi-OT model differ from Reinhart's (2004; to appear) processing explanation, which only accounts for problems in comprehension and does not predict any problems in production. Reinhart's processing account is based on the process of reference-set computation, which is a computation performed by the parser rather than the grammar. Reference-set computation involves constructing, for a given derivation, a reference set consisting of pairs of derivation and interpretation. In a second step, it is determined whether the given derivation is appropriate, or whether the pair of derivation and interpretation could be obtained more economically. If the latter is true, the given derivation is blocked. According to Reinhart (to appear), reference-set computation is necessary for the adult-like interpretation of object pronouns. Discussing other linguistic phenomena, she emphasizes that reference-set computation is not required for production because speakers already know what they want to say (Reinhart 2004, pp. 135-136). Although Reinhart does not extend her model to the use of subject pronouns that is the focus of the present study, Reinhart's model does not predict any problems with respect to production.

The production errors with subject pronouns that our model predicts, result from the non-recoverability of the intended meaning. The effect of the constraint sub-hierarchy REFERENTIAL ECONOMY is that there is a general preference for pronouns as the subject of a sentence. If a pronoun is used for referring to a topic, there is no problem. However, if a pronoun is used for referring to a non-topic, whereas a topic of the same gender is available, the intended referent is not recoverable again by the hearer. All else being equal, the hearer will prefer the topic as the referent of the pronoun and thus assign an interpretation to the pronoun which is different from the intended meaning. So using a pronoun to refer to a non-topic yields a non-recoverable meaning. To avoid non-recoverability of meanings, speakers should optimize bidirectionally and also take into account the interpretations the hearer will assign to the produced forms.

If children are unable to optimize bidirectionally, they are predicted to overuse subject pronouns and also produce subject pronouns when intending to refer to non-topics. 
Indeed, Karmiloff-Smith (1985) found this pattern of production in children until the age of 6 . The young children (from $4 ; 0$ to $5 ; 11$ years old) in her large-scale experiment involving 150 native English speaking children and 90 native French speaking children from 4 to 9 years old would typically produce strings of pronouns referring at times to the topic and at other times to a non-topic, as is illustrated by the use of the pronoun he in the following sequence:

(2) “The little boy's walking along. He's in the sunshine and he's got a hat on. The man's giving him a balloon ... a green balloon. He asks for some money so he gives him some money and then he gives him the balloon. And then he goes home to show it to his mummy. [...]."

(Karmiloff-Smith 1985, p. 71)

Only in the older age groups in the study (i.e., from the age of 6 on) were children able to block this non-adult use of pronouns in production. They then started to use definite NPs to refer to a non-topic. The pattern found by Karmiloff-Smith thus provides support for our hypothesis that the adult pattern of subject pronouns and definite NPs requires speakers to optimize bidirectionally, and that children are still unable to do so. $^{3}$

In the remainder of this article, we focus on subject pronouns and investigate whether, under certain conditions, adult language users can be shown to experience the same difficulties as Karmiloff-Smith's children when producing subject pronouns. Whether empirical confirmation can be found for our predictions with respect to the comprehension of R-expressions is left for further study.

\section{Study 1: Adults' Production of Referring Expressions}

\subsection{Method}

\subsubsection{Participants}

For experiment 1 we tested 25 elderly adults ( 8 male, 17 female) and 25 young adults (8 male, 16 female). The participants were all native speakers of Dutch. Some of them spoke a dialect. All 50 participants participated voluntarily and were not given any payment. The mean age of the elderly adults was $81 ; 7$ (range $62 ; 1-94 ; 8$ ). The mean

\footnotetext{
3 One of the reviewers wonders whether children's pattern is also predicted if children optimize bidirectionally but don't have full knowledge of the relevant constraints yet. Although constraints in OT are assumed to be universal and innate, it may be the case that Karmiloff-Smith's children haven't acquired the adult ranking of these universal constraints yet. This can easily be determined by considering the alternative constraint ranking PRONOUNS REFER TO THE TOPIC $>>$ AVOID R-EXPRESSIONS $>>$ AVOID PRONOUNS under bidirectional optimization (because the constraints AVOID R- EXPRESSIONS and AVOID PRONOUNS are part of a universal constraint sub-hierarchy, their ranking is fixed). Under this alternative constraint ranking, again the adult pattern emerges, rather than the pattern displayed by Karmiloff-Smith's children. So all possible rankings of the three constraints yield the same adult pattern of forms and meanings under bidirectional optimization. Only under unidirectional optimization do we find differences. Thus, assuming the three constraints adopted here, the pattern of acquisition with respect to subject pronouns and definite NPs cannot be explained straightforwardly by means of constraint reranking.
} 
age of the young adults was 23;2 (range 19;0-31;1). Unfortunately, the elderly adults and the young adults differed in level of education. Many of our elderly adults only received a few years of education (mean number of years of education: 9.0, range $6-16, \mathrm{SD}=3.3$, where SD stands for 'standard deviation'). In contrast, our young adults were much higher educated (mean number of years of education: 18.6, range $15-20, \mathrm{SD}=1.7$ ). Because it is very common among elderly adults in the northern region of the Netherlands to have received only a few years of education, whereas all young adults nowadays receive at least 12 years of compulsory education, we did not succeed in finding a matching group of young adults. See Sect. 3.3 for further discussion of this point.

As part of the experiment, we administered a verbal working memory test to all participants. To this end, we used the digit span test from the Wechsler Adult Intelligence Scale (WAIS). In a digit span test participants have to reproduce series of numbers which are read to them and which are increasing in length. All participants were tested on the forward and backward digit span test (in which the series of numbers have to be reproduced backward). Their total score is the sum of the score on the forward digit span test and the score on the backward digit span test (highest possible score: $17)$. The mean score of the elderly adults was $9.2(\mathrm{SD}=2.02)$. The mean score of the young adults was $13.0(\mathrm{SD}=2.29)$.

\subsubsection{Materials and Design}

To elicit the production of referring subjects we designed our own materials in a way that was inspired by Karmiloff-Smith's (1985) production study. Our elicitation study used 8 stories drawn by 5 different people. Every story consisted of 6 pictures (see Fig. 1 for an example). The structure of the stories is identical. Each story features a main character and a subsidiary character of the same gender. The main character is present on all pictures. The subsidiary character is introduced in the second picture and is also present in the third and fourth picture. The main character is the actor in all pictures but the fourth one, in which the subsidiary character is the actor.

The main character is the only referent present in the first picture and is the actor in pictures 1-3. Hence, it is the most likely initial topic of the discourse. As a result it may be referred to by the speaker by using a pronoun. However, because the subsidiary character is the actor in the fourth picture, a topic change is possible. At this point, the speaker may choose to shift from the old topic (the main character) to a new topic (the subsidiary character up to that point). The target of our production study is the action depicted in the fifth picture. If the main character is the topic of the preceding discourse, and if the participant decides on a topic shift when describing the fourth picture, it is expected that the participant will use a full NP rather than a pronoun to describe the action in the fifth picture. Because the main character and the subsidiary character are of the same gender, using a pronoun to refer to the old topic may lead to an incorrect interpretation by the hearer. So if the speaker takes into account the hearer, the speaker will use a full NP in this situation. In contrast, if the speaker does not, or cannot, take into account the hearer, the speaker will use a pronoun to describe the action depicted in the fifth picture. 


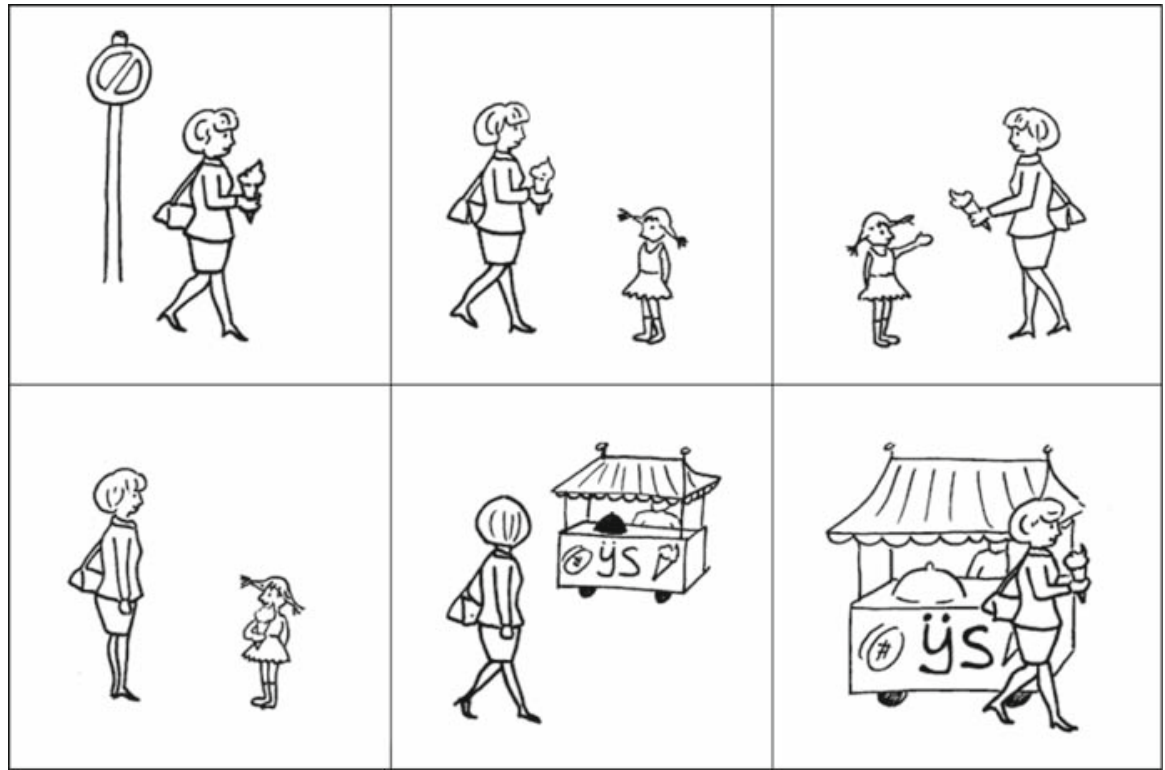

Fig. 1 One of the picture stories used

\subsubsection{Procedure}

The entire session was recorded. In addition, the responses on the target picture were scored by hand by one of the experimenters. We started the experiment with some general questions regarding age, background and level of education. Then we administered the digit span test. After the digit span test we continued with the production task, and we ended the session with a comprehension task (study 2), which is described in Sect. 4. The entire session (general questions, digit span test, production task, and comprehension task) took about one hour.

We started the production task by giving the participants instructions about the task. We told them we would show them a story made up of 6 pictures and asked them to describe each picture in one or two sentences. If the participant seemed to not understand our instructions we repeated them. Some of the elderly participants did not seem to understand that the pictures were part of a story, even after we explicitly said so. They introduced the main character again and again in subsequent pictures (e.g., "Here is a doctor. Here is a doctor who is sitting. Here is a doctor who is sawing."). In these cases we pointed out that the individual on each separate picture was the same individual throughout the story. If the participant had difficulties with word finding, we helped them. However, we never provided any help with the structure of a sentence or with a target expression.

Each picture in the production task was drawn on A4 format so they were clearly visible also to the elderly adults. The pictures were presented one by one so that the participant could see only one picture at a time and was not influenced by the previous or next picture. We turned over the pages at a regular pace so that the participant 
would not focus too much on the details in the pictures and would only describe the main action depicted. The pictures were visible to one of the experimenters only, and invisible to the other experimenter.

\subsubsection{Transcription and Coding in Study 1}

We transcribed the stories the participants produced. To analyze the data we first had to determine the initial topic of the discourse. Therefore, we looked at the utterances describing pictures $1-3$. At the point where the participant has described picture 3 , the topic of the discourse was determined on the basis of the following two criteria: The topic is the subject of the last sentence and/or the topic is pronominalized in the last sentence. In case of a conflict between these two criteria, the second criterion was taken to be decisive. That is, if the subject was a full NP and the object was pronominalized, the referent of the object pronoun was analyzed as the topic.

To analyze the data further we had to decide whether or not a topic shift has occurred in the story. A topic shift was elicited in picture 4 by changing the actor in the picture. However, we did not only look at the description of picture 4 but also at the description of picture 3 , because we noticed in our transcripts that in some cases a topic shift occurred slightly earlier, perhaps because some participants already anticipated what would happen next in the picture story. At the point where the participant has described picture 4, the topic was determined using the same criteria as mentioned earlier. If the topic at this point differed from the initial topic, we coded this as a topic shift. We only included in our further analysis elicited stories in which a topic shift occurred.

Finally, we looked at target picture 5 and scored whether, in the case of a topic shift, the action depicted by picture 5 was described using a definite NP or a pronoun for referring to the only referent present in the picture.

Although we tried to elicit a topic shift, in many cases a topic shift was avoided by the participant by using a passive construction, an impersonal sentence, or direct speech. Also, in some cases the new topic was not the subsidiary character but an object undergoing some action (e.g., the ice cream cone). As mentioned before, some participants described the pictures separately and failed to describe them as part of a story. All these cases were scored as 'other responses'.

Two of the authors separately analyzed and scored the stories. Cohen's Kappa statistic was $\kappa=.68, \mathrm{SE}=.03, p<.0001$ (Cohen 1960), a substantial agreement between the two analyses according to Landis and Koch (1977). In case of a difference between the two analyses, the results were discussed with one of the other authors, after which a decision was made.

\subsection{Results of Study 1}

\subsubsection{Production of Referring Expressions on the Basis of Target Picture}

Figure 2 shows the distribution of referring expressions produced by the young adults and the elderly adults for referring to the old topic after a topic shift has occurred. 


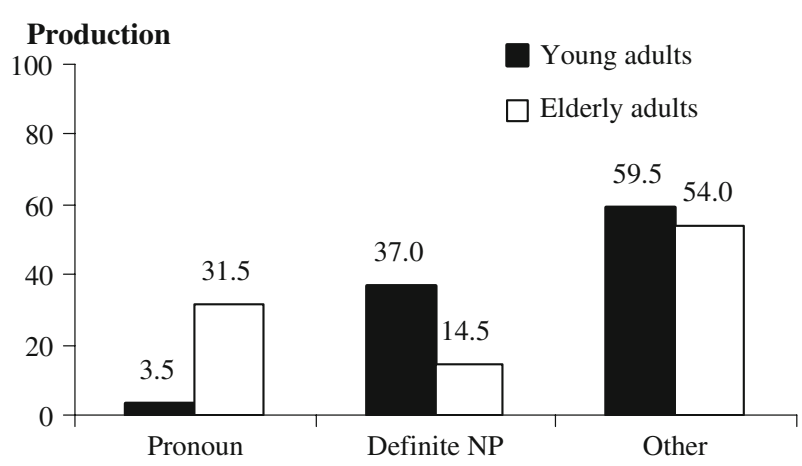

Fig. 2 Production of referring expressions

Two Repeated Measures ANOVAs were performed, one on the basis of mean percentages per participant (F1, or analysis by participants) and one on the basis of mean percentages per item (F2, or analysis by items). ${ }^{4}$ Type of Response (pronoun vs. definite NP vs. other) was treated as a within-subject and within-item factor, Age group (young vs. old) was treated as a between-subject factor in the analysis by participants, and as a within-item factor in the analysis by items. To guard against possible violations of the assumption of sphericity, the Huyn-Feldt correction was applied whenever the factor Type of Response was involved, as it has more than two levels (Stevens 1992). Original degrees of freedom will be reported.

The results of these analyses showed a significant main effect of Type of Response $(\mathrm{F} 1(2,96)=79.17 ; p<.001 ; \mathrm{F} 2(2,14)=5.63 ; p<.05)$, which was, however, qualified by a significant interaction of Age group and Type of Response $(\mathrm{F} 1(2,96)=45.34$; $p<.001 ; \mathrm{F} 2(2,14)=13.31 ; p<.01)$. Posthoc t-tests (two-tailed) revealed that this interaction was brought about by the presence of a significant difference between the age groups both with respect to mean percentage of pronouns $(\mathrm{t} 1(48)=-9.04$; $p<.001 ; \mathrm{t} 2(7)=-6.25 ; p<.001)$ and with respect to mean percentage of definite NPs $(\mathrm{t} 1(48)=5.90 ; p<.001 ; \mathrm{t} 2(7)=3.24 ; p<.05)$. There was no significant difference in 'other' responses between the two age groups (young: $\mathrm{M}=59.5 \%, \mathrm{SD}=14.6$; elderly: $\mathrm{M}=54.0 \%, \mathrm{SD}=14.8$; both $p$-values $>.20$ ). In other words, the elderly participants produced almost ten times more pronouns $(\mathrm{M}=31.5 .0 \% ; \mathrm{SD}=16.6)$ than the young participants $(M=3.5 \% ; \mathrm{SD}=6.8)$. In contrast, a substantial amount of the referring expressions produced by the young adults in this situation were definite NPs $(\mathrm{M}=37 \% ; \mathrm{SD}=13.7)$, whereas only $14.5 \%(\mathrm{SD}=11.8)$ of the referring expressions produced by the elderly adults in this situation were definite NPs. Thus, there is a substantial age group difference in the use of a pronoun or a definite NP for referring to the old topic.

In summary, then, our participants generally tried to avoid a topic shift. However, in the cases they did produce a shifted topic, the elderly participants were much more

\footnotetext{
4 The percentages of the production and comprehension task data were for analysis purposes normalized using an arcsine transformation (see http://www2.chass.ncsu.edu/garson/pa765/assumpt.htm\#transforms, last consulted 30.09.2007).
} 
likely to use a pronoun than a definite NP for referring to the old topic. Among the young participants, on the other hand, there was a strong preference to use a definite $\mathrm{NP}$, instead of a pronoun, for referring to the old topic.

\subsubsection{Correlation Between Working Memory Capacity and Pronoun Use}

We also looked at the relation between the amount of pronouns produced by the two populations and their scores on the WAIS digit span test. The mean score of the elderly population on the WAIS test was significantly lower $(\mathrm{M}=9.2, \mathrm{SD}=2.02)$ than that of the young adults $(\mathrm{M}=13.0, \mathrm{SD}=2.29), t(48)=6.22, p<.001)$. On the one hand, there is a significant negative correlation between the use of pronouns in the language production task and the scores on the memory task, Pearson $r(48)=-.33, p<.05$. The test shows that the higher the scores of the WAIS test of the age group, the fewer pronouns the group produced. On the other hand, there is a stronger positive correlation between the production of definite NPs in the production task and the scores of the WAIS test, Pearson $r(48)=.51, p<.001$.

\subsection{Discussion of Study 1}

The elderly adults in our study produced significantly more pronouns than the young adults when referring to the old topic after a topic shift has occurred. Fragment (3) shows a typical discourse (translated from Dutch) produced by one of our young participants, and fragments (4) and (5) show typical discourses produced by two of our elderly participants. All fragments are descriptions of the picture story in Fig. 1, in which the main character is a woman and the subsidiary character a girl.

(3) Young participant \# 8 (female, age 23;3): ${ }^{5}$

An, um, woman holding an ice cream cone is walking past a road sign. The woman with the ice cream cone comes across a girl. The woman gives the girl the ice cream cone. The girl is eating from the ice cream cone. Well, the woman again passes an ice cream van. The woman buys another ice cream cone.

In the discourse in (3), the woman is the initial topic. A topic shift occurs when describing picture 4 , and the girl becomes the new topic. As predicted by our bi-OT model, when describing picture 5 this young participant refers to the old topic with a definite NP ("Well, the woman again passes an ice cream van").

Fragment (4) is an example of a discourse where the elderly participant, at least initially, seems to view the pictures as separate pictures rather than as part of a story (witness the utterance "This one too", apparently referring to another lady than the one introduced in the previous utterance).

(4) Elderly participant \# 38 (female, age 83;5):

A lady, yes, is certainly holding an ice cream cone in her hand. This one too, and then that girl certainly wants that, um, that ice cream cone. Well, now she gets it.

5 We numbered the young participants from 1 to 25 , and the elderly participants from 26 to 50 . 
Tableau 2 Use of referring expressions in the description of picture 1

\begin{tabular}{lllll}
\hline & Full NP & Pronoun & Other & $\begin{array}{l}\text { Total no. of referring } \\
\text { expressions }\end{array}$ \\
\hline $\begin{array}{l}\text { Young } \\
(n=25)\end{array}$ & 199 & 0 & 1 & 200 \\
$\begin{array}{l}\text { Elderly } \\
(n=25)\end{array}$ & $(99.5 \%)$ & $(0.0 \%)$ & $(0.5 \%)$ & $(100 \%)$ \\
\hline
\end{tabular}

She enjoys it. There she is going to buy an ice cream cone. There too, at the ice cream van.

In the discourses in (4) and (5), the elderly participants refer to the old topic 'the lady' with a pronoun when describing picture 5 ("There she is going to buy an ice cream cone"; "And there she has ice cream van"), even though a topic shift has occurred and the pronoun she was used in the previous utterance to refer to the new topic 'the girl'. This overuse of pronouns resembles the pattern Karmiloff-Smith (1985) found for her youngest group of children.

(5) Elderly participant \# 39 (female, age 89;7):

Oh, he has an ice cream cone. Yes, that girl says: is that ice cream cone for me? See, she gets it. Yes, nice. She has ice cream, ice cream cone of that, um, lady. And there she has ice cream van. Do you still see them nowadays, those ice cream vans? Yes, see, so there she buys herself a new ice cream cone.

Note that the initial topic in (5) is introduced into the discourse by a pronoun ("Oh, he has an ice cream cone"). ${ }^{6}$ To see whether this was a common pattern in the production data of the elderly participants, we counted the types of referring expressions used for introducing the initial topic in the story for the young and the elderly group. We distinguished three categories: full NPs, pronouns, and other expressions. We only counted the referring expressions referring to the actor on the first picture. If the participant did not refer to this actor, and merely commented on the situation (for example, "It is raining"), we counted this as 'other'. An overview of the results can be found in Tableau 2.

The Repeated Measures ANOVAs revealed a highly significant interaction of Age group by Type of Response $(\mathrm{F} 1(2,96)=52.01 ; p<.001 ; \mathrm{F} 2(2,14)=104.17$; $p<.001)$. Posthoc analyses revealed significant age group differences for each type of response: Out of the 200 referring expressions, the elderly population produced 54 pronouns $(\mathrm{M}=27.0 \% ; \mathrm{SD}=23.6)$, as compared to none produced by the young adults $(\mathrm{t} 1(48)=-6.78, p<.001 ; \mathrm{t} 2(7)=-8.26, p<.001)$. However, the elderly participants showed a preference for introducing the initial topic of each story with a full $\mathrm{NP}(\mathrm{M}=61.5 \% ; \mathrm{SD}=27.2)$, though not as strong as the young adults $(\mathrm{M}=99.5 \%$; $\mathrm{SD}=2.5)(\mathrm{t} 1(48)=8.28, p<.001 ; \mathrm{t} 2(7)=12.41, p<.001)$. This latter difference

\footnotetext{
6 The he in this first utterance can only refer to male referents in Standard Dutch but can refer to both male and female referents in some of the dialects spoken in the northern part of the Netherlands. Indeed, participant \# 39 was a speaker of the Drenthe Low Saxon dialect.
} 
between the two groups may have to do with a difference in determining the focus of attention or establishing common ground. Perhaps the elderly participants overinterpret non-linguistic cues as signalling joint attention. However, for reasons of space we will not elaborate on this finding any further. More research is needed to establish whether the introduction of referents by means of pronouns is a real characteristic of elderly speech, and if so, whether the proposed explanation is in the right direction.

Returning to the main results of study 1 , we found that elderly adults produce significantly more pronouns than young adults when referring to the old topic after a topic shift has occurred. This is in line with our hypothesis that selecting a definite NP rather than a pronoun to refer to the old topic requires taking into account the hearer. If taking into account the perspective of the hearer as a speaker is more complex and requires more working memory capacity than simply selecting the best form from one's own perspective as a speaker, it is expected that the ability to do so decreases with age.

However, two alternative explanations of the results of study 1 are conceivable. First, as we noted in Sect. 3.1.1, the elderly participants differed from the young participants in their level of education. Perhaps level of education is crucial for the sophisticated use of referring expressions. However, Karmiloff-Smith's (1985) study showed that English children from the age of 6 on already produce pronouns in a more adult way and hardly produce any non-recoverable pronouns anymore. If level of education were the crucial factor, we would expect our elderly participants to perform at least as well as Karmiloff-Smith's oldest group of children, which is not the case.

A second alternative explanation of the results of study 1 is that the elderly participants in our study show early signs of Alzheimer. Almor et al. (1999) found that their 11 Alzheimer patients (mean age 80.7; range 64-89) produce more pronouns than their 9 healthy elderly controls (mean age 77; range 70-85) in their spontaneous speech. However, none of our 25 elderly participants (mean age 81.6; range 62-95) was diagnosed with Alzheimer or any other degenerative cognitive disease. All of them were still living independently and did not need any special care. To find out whether our elderly participants produced more pronouns in general (rather than only in utterances following a topic shift) than our young participants, we counted all nominal expressions used by the participants in their description of pictures 1-3 (i.e., before the potential topic shift). We then scored the instances of nominal reference by means of a pronoun. An overview of the results can be found in Tableau 3.

Out of the nominal references produced by our elderly participants, almost half $(\mathrm{M}=44 \%$; $\mathrm{SD}=7.9)$ were pronouns, compared to $23 \%$ pronouns $(\mathrm{SD}=9.5)$ produced by the young adults. This group difference was significant $t(42)=-7.56, p<.001$. Thus, the elderly participants used almost twice as many pronouns as the young participants. There is a slight numerical difference between the total numbers of nominal referring expressions used by the different populations. On average, the elderly participants produced slightly more nominal references $(\mathrm{M}=87, \mathrm{SD}=44)$ than the young participants did $(\mathrm{M}=84, \mathrm{SD}=25)$. However, this difference was not significant $(p>.78)$.

So we do find that the elderly participants use significantly more pronouns in general in their narratives than the young participants. However, this general tendency does not entirely explain the excessive use of pronouns for reference to the old topic 


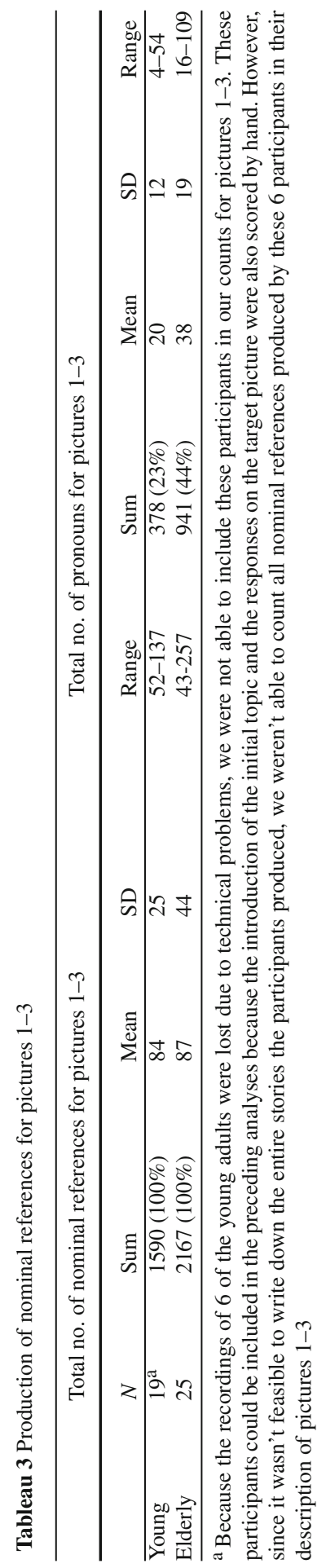


following a topic shift in the elicited production task. Although the elderly adults in general produced almost twice as many pronouns as the young adults did (which is partly caused by their tendency to use a pronoun for introducing the initial topic), after a topic shift they produced pronouns almost ten times more often than the young adults did. Thus there is a clear difference between young and elderly adults in their choice of a referring expression for the old topic, which cannot be explained by general preferences.

\section{Study 2: Adults' Comprehension of Referring Expressions}

\subsection{Method}

\subsubsection{Participants}

In study 2 all participants of study 1 participated. Study 1 and study 2 were administered in one and the same session.

\subsubsection{Materials and Design}

In this study we investigated the comprehension of pronouns. The materials were designed in such a way that the written stories were maximally similar to the picture stories of study 1 . Our comprehension study consisted of 8 written stories. All stories were made up of 6 sentences. The structure of all written stories is identical. Each story features a main character (the topic) and a subsidiary character (a nontopic) of the same gender. The main character is introduced with a proper name in the first sentence and referred to by a subject pronoun in the next two sentences. The subsidiary character is introduced with a proper name as the direct object or prepositional object in the second sentence. This subsidiary character is the non-pronominal subject of the fourth sentence, and carries the thematic role of agent. Sentence 5 and 6 have a pronoun in subject position. Figure 3 presents an example of a written story.

Because the two characters are of the same gender, the pronouns in sentences 5 and 6 can in principle refer to both characters. Both options are equally plausible. Consequently, the participants have to take into account the preceding discourse structure to determine the reference of the pronouns. The participant can either take the main character (the old topic) to be the referent of the subject pronoun, or the subsidiary character. Assuming that pronouns preferably refer to topics, in the latter case a topic shift has occurred such that the subsidiary character has become the new topic.

\subsubsection{Procedure}

We told the participants they would hear or read 8 written stories. We asked them if we had to read the story to them or if they wanted to read the story themselves. Furthermore, we told the participants that we would ask them a question after each story. Which answer should be given to the question is dependent on the resolution 
1. Piet werkt vandaag in de tuin want het is prachtig weer.

2. Hij wiedt eerst het onkruid met zijn kleinzoon Joost.

3. Daarna maait hij het gras met zijn nieuwe grasmaaier.

4. Joost helpt met de planten water geven.

5. Hij heeft hard gewerkt vandaag en is nu best wel moe.

6. Toch moet hij ook nog de kippen en de konijnen voeren.

Comprehension question: Wie moet de kippen en de konijnen nog voeren?

1. Today, Piet is working in the garden because the weather is very nice.

2. With his grandson Joost, he first weeds the garden.

3. Then he mows the lawn with his new lawn mower.

4. Joost helps watering the plants.

5. He has worked hard today and now feels a bit tired.

6. Nevertheless he still has to feed the chickens and the rabbits.

Comprehension question: Who still has to feed the chickens and the rabbits?

Fig. 3 One of the written stories used

of the pronoun in sentence 6. If the pronoun is resolved as the old topic, the expected answer is the old topic. If the pronoun is resolved as the shifted topic, the expected answer is the shifted topic. We emphasized that there was no right or wrong answer to the question.

\subsubsection{Transcription and Coding in Study 2}

The comprehension data where analyzed by scoring how often participants answered by mentioning the main character (the old topic) or the subsidiary character (the shifted topic). No other answers were given by any of the participants.

\subsection{Results of Study 2}

Figure 4 gives an overview of the results of the comprehension task. It suggests that there is almost no difference in the interpretation of the pronoun in the final sentence of the stories between the two populations. The distribution of the two possible answers (reference to the main character or to the subsidiary character) seems almost equal in the two populations. Indeed, Repeated Measures ANOVAs with Type of Topic (old vs. shifted) as a within-subjects and within-items factor, and Age group as a between-subject and within-item factor did not produce significant results (all $p$-values $>.33$ ). Both populations thus had no clear preference for reference by the pronoun to the subsidiary character (the shifted topic) in the final sentence of the story. 


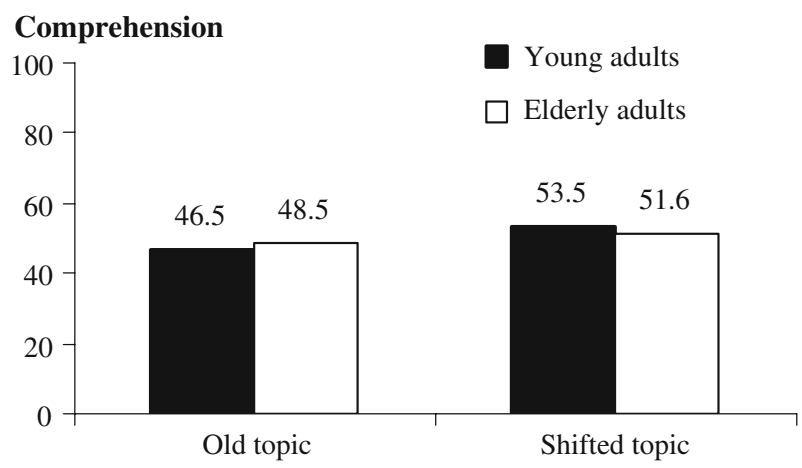

Fig. 4 Comprehension of pronouns

\subsection{Discussion of Study 2}

The elderly participants in our study did not differ significantly from the young participants with respect to their responses on the comprehension task. On average, participants interpreted the pronoun as referring to the main character in half of the cases and as referring to the subsidiary character in the other half of the cases. In terms of discourse structure, participants interpreted half of the stories as involving a continuing topic and the other half of the stories as involving a topic shift. The subsidiary character was introduced as a direct object or prepositional object in the second sentence and was the agentive subject of the fourth sentence. Apparently, this was not sufficient to increase the salience of the subsidiary character so that a topic shift was unavoidable.

When comparing the patterns of results between the different items, it appeared that some of the stories did elicit a clear preference for a continuing topic, whereas others elicited a clear preference for a topic shift. Since the discourse structures of the stories were identical, these preferences may be due to subtle effects of world knowledge or plausibility. Importantly, for all but one story the preferences of the young adults and the elderly adults were the same. Almor et al. (1999), in their study of Alzheimer patients, found that the Alzheimer patients not only experienced production problems (as witnessed by their excessive use of pronouns) but also experienced comprehension problems. In particular, the Alzheimer patients showed slower comprehension when pronouns were used, but faster comprehension when full NPs were used, regardless of their discourse status. Our elderly participants, in contrast, gave similar responses as the young participants on the off-line comprehension task in study 2. Whether they would also give similar responses as the young participants on an on-line task, however, remains to be seen.

\section{General Discussion}

The observed pattern of deficient production of subject pronouns in elderly adults coupled with their unimpaired comprehension suggests that producing a definite NP to 
refer to a non-topic requires that the general preference for a pronoun must be actively blocked through bidirectional optimization. Only through bidirectional optimization is it possible to check whether a selected expression is recoverable for a hearer. Because bidirectional optimization requires sufficient speed of processing and working memory capacity, it is not yet developed in young children until at least the age of $6 .{ }^{7}$ Due to their decreasing speed of processing and working memory capacity, bidirectional optimization becomes difficult again in elderly adults. The observed difference in performance between young and elderly adults thus supports the view that, when the discourse context is relevant, bidirectionally optimal pairs have to be computed online and cannot be part of the grammar as the fossilized results of principles of language use (contra Blutner and Zeevat 2004).

We argued in Sect. 2 that a bi-OT analysis is able to explain elderly adults' problems in production (study 1 ) as well as children's late comprehension delays (de Hoop and Krämer 2005/2006; Hendriks and Spenader 2004, 2005/2006) and late production delays (cf. Karmiloff-Smith 1985). If a bi-OT analysis indeed provides the best explanation of these data, then our analysis would account for a wider range of observations than Reinhart's (2004, to appear) processing account and Thornton and Wexler's (1999) pragmatic account, which only explain children's late comprehension delays. Neither Reinhart's processing account nor Thornton and Wexler's pragmatic account predicts any problems in pronoun production. However, as one of the reviewers pointed out, an alternative explanation of the data is suggested by Zeevat (2000). Zeevat argues that bi-OT grammars must be asymmetrical. In the OT model he proposes, speakers optimize unidirectionally and only consider their own perspective when speaking, whereas hearers optimize bidirectionally and take into account the perspective of the speaker when selecting the optimal meaning. But if we take the results of the present study seriously, a different type of asymmetrical grammar than the one proposed by Zeevat is required to explain our data. As we showed, young adult speakers seem to take into account the perspective of the hearer. While this may be true, perhaps young adult hearers do not take into account the perspective of the speaker. This type of asymmetrical bi-OT grammar (i.e., the opposite model of the one proposed by Zeevat, with speakers optimizing bidirectionally and hearers optimizing unidirectionally) is employed by Wilson (2001) and Jäger (2004). In such an asymmetrical bi-OT grammar, children's errors in interpreting object pronouns, and children's and elderly adults' errors in producing subject pronouns, should receive different explanations: Whereas a bidirectional account such as the one presented here may explain children's

\footnotetext{
7 Wittek and Tomasello (2005) argue that already children as young as 2.5 are sensitive to the hearer's knowledge in production and select the appropriate referring expression accordingly. The choice children were faced with in their experiments was different from the choice in Karmiloff-Smith's (1985) study and our elicitation task in study 1, though: In response to various questions, some of which introduced a referent (e.g., "Where is the broom?" vs. "What do we need?"), children either used a pronoun or null reference to refer to this previously mentioned referent, or used an indefinite noun phrase to introduce a new referent. Wittek and Tomasello's study thus seems to show that young children are sensitive to whether or not an entity has already been mentioned in the linguistic discourse (cf. also Matthews et al. 2006), which is a much simpler task than the bidirectional optimization task of taking into account the interpretation a hearer will assign to a preferred form and actively blocking this preferred form in case of a mismatch between the intended meaning and the hearer's interpretation.
} 
and elderly adults' errors in producing subject pronouns, a unidirectional explanation should be sought for children's errors in interpreting object pronouns.

As we mentioned in Sect. 1, due to their frequent use in the same fixed context, certain associations between forms and meanings can become automatic or 'fossilized' and need not be computed anew on later occasions. For this reason, it is not possible to decide between a unidirectional and a bidirectional account of children's errors in pronoun interpretation by studying the interpretation of object pronouns in elderly adults. Because the interpretation of object pronouns is a sentence-internal phenomenon, we expect it to have become automatic in adults. Once it has become automatic, it should be independent of variation in processing resources. Thus, elderly adults are predicted to make as little errors as young adults when interpreting object pronouns.

Although the present study does not shed any light on the interpretation of object pronouns directly, it does so indirectly. The bidirectional analysis of Hendriks and Spenader (2004, 2005/2006) uses Burzio's (1998) constraint sub-hierarchy REFERENTIAL ECONOMY, which expresses a preference for reflexives over pronouns, and of pronouns over full NPs. The present study yields independent support for the preference of pronouns over full NPs, because pronouns seem to be the preferred form in the absence of sufficient processing resources. Thus, the present study provides independent support for the constraint sub-hierarchy REFERENTIAL ECONOMY employed by Hendriks and Spenader in their bidirectional account of object pronouns.

The results of this study may also add to the much wider debate on whether speaking and listening in general are bilateral processes, or whether they are essentially egocentric processes. According to Clark and his colleagues (Clark and Wilkes-Gibbs 1986; Clark and Krych 2004), speaking is a bilateral process in which speakers not only monitor and take into account their own actions, but also the actions of their hearers. Evidence for speaking as a bilateral process comes from, among other things, the observation that speakers often alter the course of their utterances in response to mid-utterance reactions from the hearer. An opposite approach is advocated by Keysar and colleagues (Epley et al. 2004; Keysar 2007; Keysar et al. 1998), who argue on the basis of empirical evidence derived from perspective taking tasks that language users in general are egocentric. The difference between adults and children is a matter of degree, they claim. In comprehension, adults start out with an egocentric interpretation, like children, but adults are better able to correct this initial interpretation when necessary. Crucially, as Keysar (2007, p. 72) argues, speaker's and hearer's "consideration of the mental state of the other is not done systematically".

Our study suggests a third position, which is slightly different from both the bilateral and the egocentric approach: Language users start out with unidirectional optimization, which is an egocentric process. However, whenever language users possess sufficient processing capacities to optimize bidirectionally and take into account the opposite perspective in communication as well, they will do so. As a result, this bidirectional process may in many cases have become automatic for mature language users, as in the case of the interpretation of object pronouns. In other cases, such as the production of subject pronouns, which is highly dependent on discourse context, this bidirectional process is still effortful and affected by variation in processing resources. But when speakers and hearers take into account the opposite perspective, they do so in a 
systematic way because bidirectional optimization is driven by the properties of the grammar. Crucially, the opposite perspective is not dependent on the actual knowledge of the other, but rather on the knowledge the speaker would have if she were a hearer, and the knowledge the hearer would have if he were a speaker. So under a bidirectional account, mature language users take into account the opposite perspective, but in a highly idealized way, which may cause miscommunication if the other does not behave in this idealized way. Thus our study suggests that mature language use is a partly automatic, yet highly systematic, bilateral process built on top of the initial egocentric processes of speaking and hearing.

\section{Conclusion}

In our elicitation study, we found that elderly adults produce pronouns significantly more often than young adults when referring to the old topic in the presence of a new topic. In comprehension, no significant differences were found between elderly and young adults. There was an inverse correlation between the number of pronouns produced and working memory capacity of the population (as measured by digit span tests). These results support the hypothesis that speakers take into account hearers when selecting a referring expression, which can be formalized as bidirectional optimization in the framework of Optimality Theory. If the speaker has reasons to believe the hearer will not be able to recover a pronoun, the speaker will use a full NP instead. This pattern was confirmed by the production results of the young adults. A comparison of the production results of the young adults and the elderly adults in our study suggests that in certain cases (viz. when the previous linguistic discourse is relevant) bidirectional optimization has to be performed online. Because elderly adults are more limited in their processing capacities than young adults, they find it more difficult to optimize bidirectionally and to take into account the hypothetical hearer when selecting a referring expression. As a result, they will produce pronouns even when a pronoun will lead to a non-intended interpretation for a hearer.

Acknowledgements This investigation was supported in part by grants from the Netherlands Organisation for Scientific Research, NWO (Grants no. 277-70-005 and 015-001-103 for Petra Hendriks). The authors thank Reinhard Blutner, two anonymous reviewers, the participants of the workshop "Formal models for real people", September 2006, Amsterdam, and the participants of the Conference on Intersentential Pronominal Reference in Child and Adult Language, December 2006, Berlin, for their valuable suggestions and comments.

Open Access This article is distributed under the terms of the Creative Commons Attribution Noncommercial License which permits any noncommercial use, distribution, and reproduction in any medium, provided the original author(s) and source are credited.

\section{References}

Almor, A., Kempler, D., MacDonald, M.C., Andersen, E.S., \& Tyler, L.K. (1999). Why do Alzheimer patients have difficulty with pronouns? Working memory, semantics, and reference in comprehension and production in Alzheimer's disease. Brain and Language, 67, 202-227.

Ariel, M. (1988). Referring and accessibility. Journal of Linguistics, 24, 65-87.

Ariel, M. (1990). Anaphoric antecedents. London: Croom Helm. 
Blutner, R. (2000). Some aspects of optimality in natural language interpretation. Journal of Semantics, 17, $189-216$.

Blutner, R. (2007). Optimality theoretic pragmatics and the explicature/implicature distinction. In N. Burton-Roberts (Ed.), Advances in pragmatics (pp. 67-89). Houndmills, Basingstoke, Hampshire: Palgrave/MacMillan.

Blutner, R., de Hoop, H., \& Hendriks, P. (2006). Optimal communication. Stanford: CSLI Publications.

Blutner, R, \& Zeevat, H. (2004). Editors' introduction: Pragmatics in Optimality Theory. In R. Blutner, \& H. Zeevat (Eds.), Optimality theory and pragmatics (pp. 1-24). Houndmills, Basingstoke, Hampshire: Palgrave/Macmillan.

Bouma, G. J. (2008). Starting a sentence in Dutch: A corpus study of subject- and object-fronting. PhD dissertation, University of Groningen.

Burzio, L. (1998). Anaphora and soft constraints. In P. Barbosa, D. Fox, P. Hagstrom, M. McGinnis, \& D. Pesetsky (Eds.), Is the best good enough? Optimality and competition in syntax (pp. 93-113). Cambridge, MA: MIT Press.

Chien, Y.-C., \& Wexler, K. (1990). Children's knowledge of locality conditions on binding as evidence for the modularity of syntax and pragmatics. Language Acquisition, 13, 225-295.

Clark, H. H., \& Krych, M. A. (2004). Speaking while monitoring addressees for understanding. Journal of Memory and Language, 50, 62-81.

Clark, H. H., \& Wilkes-Gibbs, D. (1986). Referring as a collaborative process. Cognition, 22, 1-39.

Cohen, J. (1960). A coefficient of agreement for nominal scales. Educational and Psychological Measurement, 20, 37-46.

De Hoop, H., \& Krämer, I. (2005/2006). Children's optimal interpretations of indefinite subjects and objects. Language Acquisition, 13, 103-123.

Epley, N., Morewedge, C.K., \& Keysar, B. (2004). Perspective taking in children and adults: Equivalent egocentrism but differential correction. Journal of Experimental Social Psychology, 40, 760-768.

Givón, T. (1993). English grammar: A function-based introduction. Amsterdam: John Benjamins.

Grice, H. P. (1975). Logic and conversation. In P. Cole, \& J. Morgan (Eds.), Syntax and semantics (pp. 41-58). Vol. 3, Academic Press.

Gundel, J., Hedberg, N., \& Zacharski, R. (1993). Cognitive status and the form of referring expressions in discourse. Language, 69, 274-307.

Hendriks, P., \& Spenader, J. (2004). A bidirectional explanation of the pronoun interpretation problem. In P. Schlenker, \& E. Keenan (Eds.), Proceedings of the ESSLLI '04 Workshop on Semantic Approaches to Binding Theory. Nancy, France.

Hendriks, P., \& Spenader, J. (2005/2006). When production precedes comprehension: An optimization approach to the acquisition of pronouns. Language Acquisition, 13, 319-348.

Hendriks, P., van Rijn, H., \& Valkenier, B. (2007). Learning to reason about speakers' alternatives in sentence comprehension: A computational account. Lingua, 117, 1879-1896.

Jäger, G. (2004). Learning constraint sub-hierarchies: The bidirectional gradual learning algorithm. In R. Blutner, \& H. Zeevat (Eds.), Optimality theory and pragmatics (pp. 251-287). Houndmills, Basingstoke, Hampshire: Palgrave/Macmillan.

Karmiloff-Smith, A. (1985). Language and cognitive processes from a developmental perspective. Language and Cognitive Processes, 1, 61-85.

Kemtes, K. A., \& Kemper, S. (1999). Aging and resolution of quantifier scope. Journal of Gerontology: Psychological Sciences, 54B, P350-P360.

Keysar, B. (2007). Communication and miscommunication: The role of egocentric processes. Intercultural Pragmatics, 4, 71-84.

Keysar, B., Barr, D. J., \& Horton, W. S. (1998). The egocentric basis of language use: Insights from a processing approach. Current Directions in Psychological Science, 7, 46-50.

Landis, J. R., \& Koch, G. G. (1977). The measure of observer agreement for categorial data. Biometrics, $33,159-174$.

Matthews, D., Lieven, E., Theakston, A., \& Tomasello, M. (2006). The effect of perceptual availability and prior discourse on young children's use of referring expressions. Applied Psycholinguistics, 27, $403-422$.

Prince, A., \& Smolensky, P. (2004). Optimality theory: Constraint interaction in generative grammar. Blackwell. Also appeared as Technical Report CU-CS-696-93, Department of Computer Science, University of Colorado at Boulder, and Technical Report TR-2, Rutgers Center for Cognitive Science, Rutgers University, New Brunswick, NJ, April 1993. 
Reinhart, T. (2004). The processing cost of reference-set computation: Acquisition of stress shift and focus. Language Acquisition, 12, 109-155.

Reinhart, T. (to appear). Processing or pragmatics? Explaining the coreference delay. In T. Gibson and N. Pearlmutter (Eds.), The processing and acquisition of reference. Cambridge, MA: MIT Press.

Stevens, J. (1992). Applied multivariate statistics for the social sciences. Hillsdale, NJ: Lawrence Erlbaum. Thornton, R., \& Wexler, K. (1999). Principle B, VP ellipsis and interpretation in child grammar. Cambridge, MA: MIT Press.

Wilson, C. (2001). Bidirectional optimization and the theory of anaphora. In G. Legendre, J. Grimshaw, \& S. Vikner (Eds.), Optimality-theoretic syntax (pp. 465-507). Cambridge, MA: MIT Press.

Wingfield, A., Stine, E. A. L., Lahar, C. J., \& Aberdeen, J. S. (1988). Does the capacity of working memory change with age? Experimental Aging Research, 14, 103-107.

Wittek, A., \& Tomasello, M. (2005). Young children's sensitivity to listener knowledge and perceptual context in choosing referring expressions. Applied Psycholinguistics, 26, 541-558.

Zeevat, H. (2000). The asymmetry of optimality theoretic syntax and semantics. Journal of Semantics, 17, 243-262. 